\section{Fistula from an internal iliac artery aneurysm to the sigmoid colon after endovascular arterial repair}

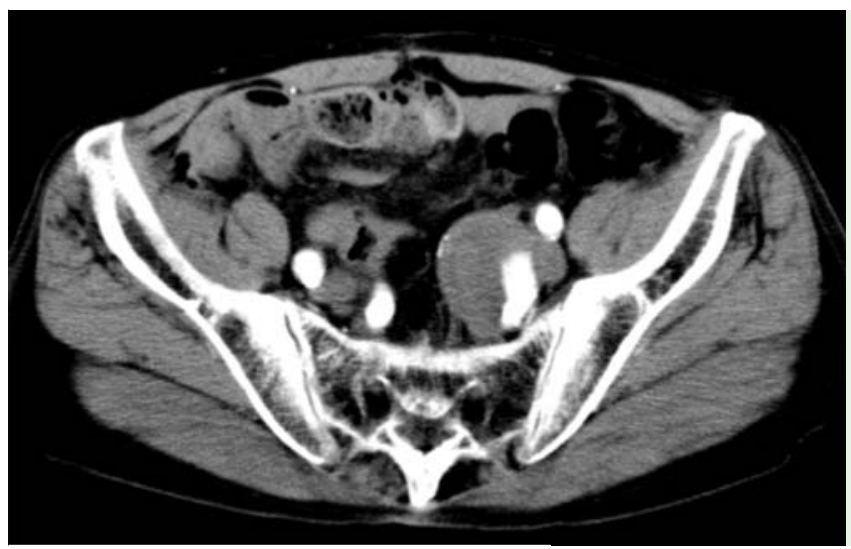

Fig. 1 Contrast-enhanced computed tomography showed a $5 \mathrm{~cm}$ in diameter internal iliac artery aneurysm prior to endovascular arterial repair.

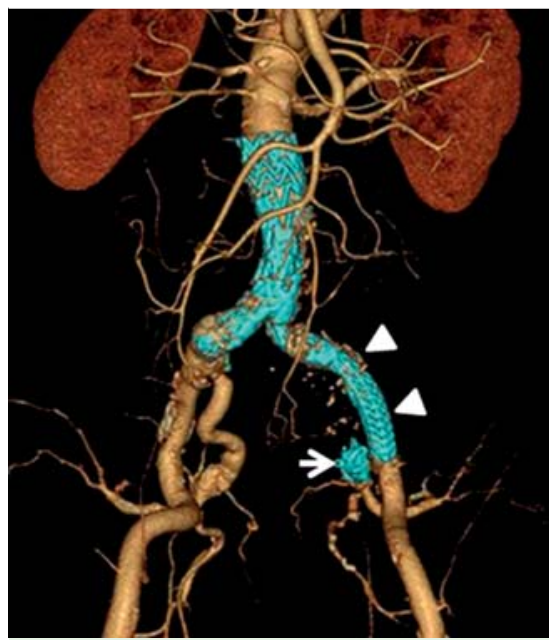

Fig. 2 Three-dimensional computed tomography angiography after endovascular arterial repair indicated exclusion of the internal iliac artery aneurysm by coil embolization (arrow) and stent grafting (arrowhead).
An 82-year-old patient presented with a history of fever for 2 weeks and occasional rectal bleeding. He had undergone endovascular repair of a left internal iliac artery aneurysm (IIAA) 3 years previously ( Fig. 1); distal occlusion of the IIAA with coil embolization and proximal occlusion using an aorto-external iliac artery stent graft achieved successful exclusion of the IIAA ( Fig.2). Contrast-enhanced computed tomography showed an IIAA (diameter, $6 \mathrm{~cm}$ ) without obvious enhancement but with massive air in the aneurysm ( Fig.3). Colonoscopy revealed an ulcer (diameter, $3 \mathrm{~cm}$ ) with a base covered with feces, approximately $20 \mathrm{~cm}$ from the anal verge ( Fig.4). Gastrografin enema indicated a fistula from the sigmoid colon to the IIAA ( $\bullet$ Fig.5). The patient underwent colostomy, result-

ing in relief of symptoms including fever and rectal bleeding, and rapid decrease in the size of the IIAA, which finally disappeared.

Aneurysms of the internal iliac artery are rare, representing only $1 \%$ of all cases of aortoiliac aneurysmal disease [1]. Rupture of an IIAA to adjacent organs, including the extremely rare complication of rupture into the rectosigmoid colon, is associated with high mortality [2,3]; for this reason, prophylactic repair is recommended for IIAAs more than $3 \mathrm{~cm}$ in diameter. Traditionally, open surgery was used for IIAA repair; however, open surgery is associated with high morbidity and mortality rates. Recently, endovascular repair of IIAA, including by stent grafting, various embolization techniques, or a combination of both, is being increasingly performed in clinical practice as a novel, less invasive treatment for these aneurysms. However, the long-term complications of endovascular repair of IIAA remain unclear $[4,5]$. The case reported here represents a rare, unusual complication of an IIAA-colon fistula after endovascular repair. Even if an IIAA is excluded by endovascular repair, if the patient subsequently presents with fever and/or rectal bleedings and - particularly if the aneurysm has not decreased in size since the repair - physicians should be aware that an IIAA-colon fistula may be present.

\title{
Endoscopy_UCTN_Code_CCL_1AF_2AH
}

\section{Competing interests: None}
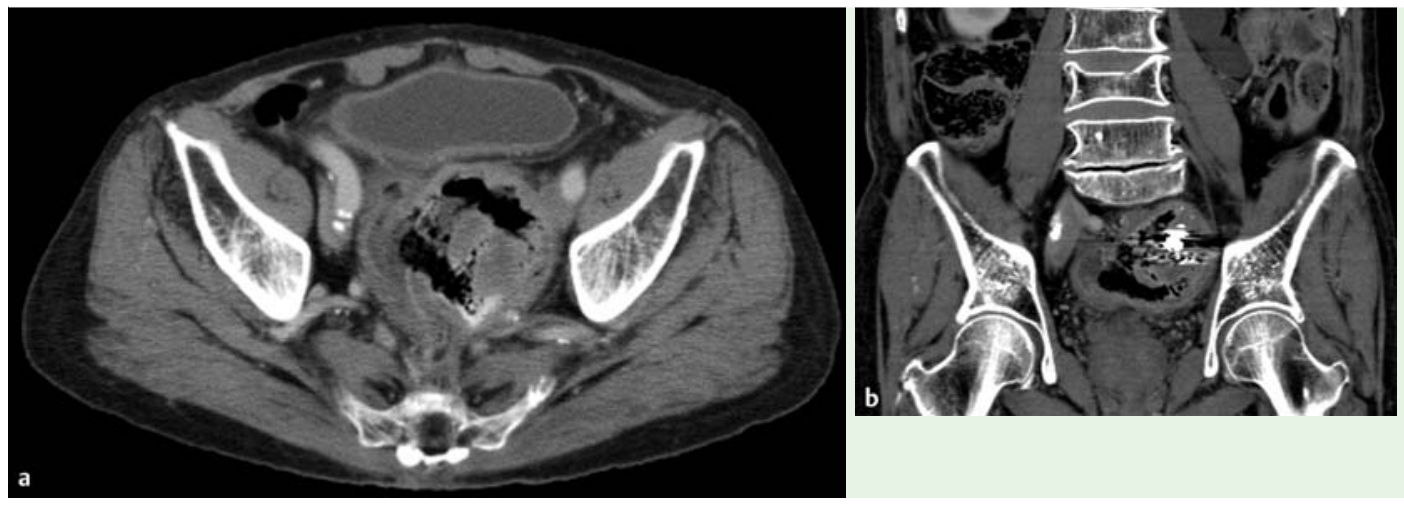

Fig. 3 Contrast-enhanced computed tomography indicated the presence of abundant air in the aneurysm, which was close to the sigmoid colon. a Axial view, $\mathbf{b}$ coronal view. 


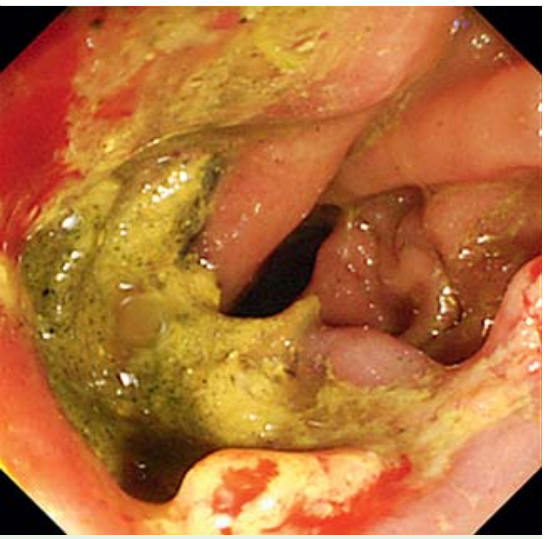

Fig. 4 Colonoscopy revealed an ulcer with a diameter of $3 \mathrm{~cm}$, the base of which was covered with feces.

\section{Akira Goto ${ }^{1}$, Ishimine Yu' ${ }^{1}$, Takafumi Naito², Takashi Yabana', Takeya Adachi $^{1}$, Yoshihiro Kondo ${ }^{1}$}

${ }^{1}$ Department of Gastroenterology, Otaru General Hospital, Otaru, Japan

2 First Department of Internal Medicine, Sapporo Medical University, Sapporo, Hokkaido, Japan

\section{References}

1 Brunkwall J, Hauksson $\mathrm{H}$, Bengtsson $\mathrm{H}$ et al. Solitary aneurysms of the iliac arterial system: An estimate of their frequency of occurrence. J Vasc Surg 1989; 10: 381-384

2 Katoh J, Shindo S, Kina S et al. Rupture of an isolated internal iliac artery aneurysm into

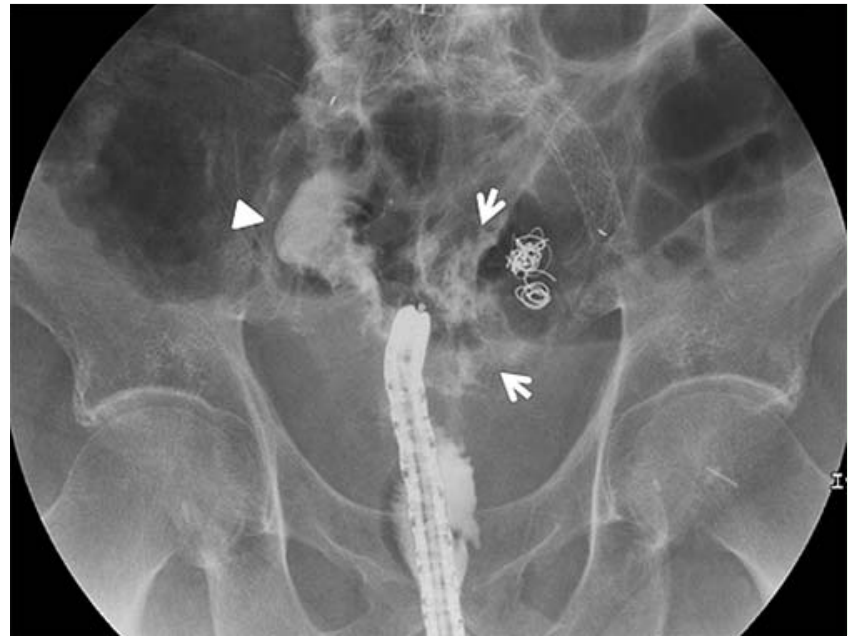

Fig. 5 Gastrografin enema showed a fistula between the aneurysm (arrows) and the sigmoid colon (arrowhead).

the rectum: report of a case. Surg Today 1995; 25: 554-556

3 Karkos $C D$, Oshodi TO, Vimalachandran D et al. Internal iliac aneurysm rupture into the rectum following endovascular exclusion: an unusual cause of massive lower gastrointestinal bleeding. J Endovasc Ther 2002; 9: 907-911

4 Eggebrecht $H$, Mehta $R H$, Dechene A et al. Aortoesophageal fistula after thoracic aortic stent-graft placement: a rare but catastrophic complication of a novel emerging technique. JACC Cardiovasc Interv 2009; 2: 570-576

5 Millon A, Paquet Y, Ben Ahmed $S$ et al. Midterm outcomes of embolisation of internal iliac artery aneurysms. Eur J Vasc Endovasc Surg 2013; 45: 22-27

\section{Bibliography}

DoI http://dx.doi.org/

10.1055/s-0034-1377354

Endoscopy 2014; 46: E367-E368

(c) Georg Thieme Verlag KG

Stuttgart · New York

ISSN 0013-726X

\section{Corresponding author}

\section{Akira Goto, MD, PhD}

Department of Gastroenterology

Otaru General Hospital

1-2-1, Wakamatsu

Otaru 047-8550

Japan

Fax: +81-134-326424

goa@sapmed.ac.jp 